\title{
Interactions between Adjacent Ganglia Bring About the Bilaterally Alternating Differentiation of RAS and CAS Neurons in the Leech Nerve Cord
}

\author{
Seth S. Blair, ${ }^{a}$ Mark Q. Martindale, ${ }^{b}$ and Marty Shankland \\ Department of Anatomy and Cellular Biology, Harvard Medical School, Boston, Massachusetts 02115
}

\begin{abstract}
Antibodies to small cardioactive peptide (SCP) label a segmentally iterated subset of cells in the leech nerve cord, including the previously identified alternating SCP (AS) neurons. Unlike the majority of leech neurons, these cells are asymmetrically distributed in the adult nerve cord. Moreover, each AS neuron shows a strong tendency to lie on alternate right and left sides in successive ganglia. Previous work has shown that these unpaired neurons arise from bilaterally paired embryonic homologues, only 1 of which takes on the mature immunoreactive phenotype. The 2 AS homologues within a ganglion compete for this fate, in that either the right or the left homologue will become a mature AS neuron with a high degree of reliability if its contralateral homologue is ablated during embryogenesis. In this paper, we demonstrate the existence of interactions between neurons in adjacent ganglia that could account for the alternation of sides observed during normal development. The unilateral ablation of a single AS homologue neuron forced its contralateral homologue to take on the mature AS fate, and this consistently biased the side of AS development in adjacent, unlesioned ganglia both anterior and posterior to the lesion. One of the AS neurons, the caudal alternating SCP (CAS) cell, was injected with Lucifer yellow in adult nerve cords and was shown to have a large primary axon that extends into more anterior ganglia, as well as other, finer axons that are variable in number and arrangement. If the interganglionic interaction of AS neuron homologues is mediated by their primary axons, signals of developmental import must be transmitted both anterogradely and retrogradely along the axon's length. The present results indicate that the development of individual AS neurons is influenced by homologous cells located in the same and neighboring ganglia and suggest that the final, multisegmental patterning of the AS neuron distribution is not predetermined, but rather, arises as an emergent property of the cell interactions that occur during nervous system differentiation.
\end{abstract}

\footnotetext{
Received Apr. 5, 1990; revised May 22, 1990; accepted Jun 6, 1990.

This work was supported by March of Dimes Basil O' Connor Award 5-593 and Research Grant 1-1190, as well as by National Science Foundation Research Grant BNS-8718045. M.Q.M. was supported by NIH Fellowship F32 GM12481, and M.S. was an Alfred P. Sloan Research Fellow.

Correspondence should be addressed to Marty Shankland, Department of Anatomy and Cellular Biology, Harvard Medical School, Boston, MA 02115.

a Present address: Department of Zoology, University of Wisconsin, Madison, WI 53706

b Present address: Department of Organismal Biology and Anatomy, University of Chicago, 1025 East 57 th Street, Chicago, IL 60637.

Copyright (c) 1990 Society for Neuroscience $0270-6474 / 90 / 103183-11 \$ 03.00 / 0$
}

The fundamental organization of the leech nerve cord is bilaterally symmetric. The central neurons develop from bilaterally paired embryonic precursor cells (Whitman, 1878; Weisblat et al., 1980; Blair, 1983; Kramer and Weisblat, 1985; Weisblat and Shankland, 1985), and the majority of the neurons that have been identified are encountered as functionally equivalent bilateral pairs (Ort et al., 1974). However, the leech nervous system also contains a small number of "unpaired" neurons, called such because they are without any obvious contralateral homologue in the adult. Those unpaired neurons whose development has been studied in detail do in fact arise from bilaterally paired embryonic neurons, but become distinct from their contralateral homologues by a process of asymmetric differentiation or survival (Macagno and Stewart, 1987; Stuart et al., 1987; Shankland and Martindale, 1989). All of the unpaired leech neurons that have been characterized to date can arise with equal probability in any given segment from the right or left side of the embryonic germinal plate. In this paper, we investigate the neuronal interactions that influence this decision and coordinate the spatial patterning of these asymmetries over multiple segments.

The neurons examined in this paper are the unpaired rostral and caudal alternating SCP neurons (individually, RAS and CAS neurons, respectively, or collectively, the AS neurons). The RAS and CAS neurons show many similarities in their development, morphology, and biochemical differentiation, though they arise from distinct embryonic blastomeres: the $\mathrm{N}$ and $\mathrm{M}$ teloblasts, respectively (Shankland and Martindale, 1989). These AS neurons are among a small set of leech neurons that stain both with a monoclonal antibody to the molluscan SCP and with antisera to phenylalanine-methionine-arginine-phenylalanine amide (FMRFamide), and their distribution has been described for several different species of leech (Evans and Calabrese, 1989; Shankland and Martindale, 1989). As shown in Figure 1, each of the 2 cells is restricted to a specific segmental domain. In adult leeches of the species considered here, the RAS neuron is found in $\mathbf{4}$ adjacent rostral segments, specifically, the last neuromere of the fused subesophageal ganglion (S4) and the first 3 abdominal segments (A1-A3). In contrast, the CAS neuron is only found in the 4 most caudal abdominal segments (A18A21) and at least the first 4 segments of the fused tail brain (T1T4). Both AS neurons have cell bodies located laterally within the segmental ganglion on their side of origin and do not have obviously immunoreactive contralateral homologues within that same segmental ganglion. While the immunoreactive AS neurons can lie on either side, they tend to alternate from right to left in successive ganglia with a high degree of fidelity ( $>95 \%$ 


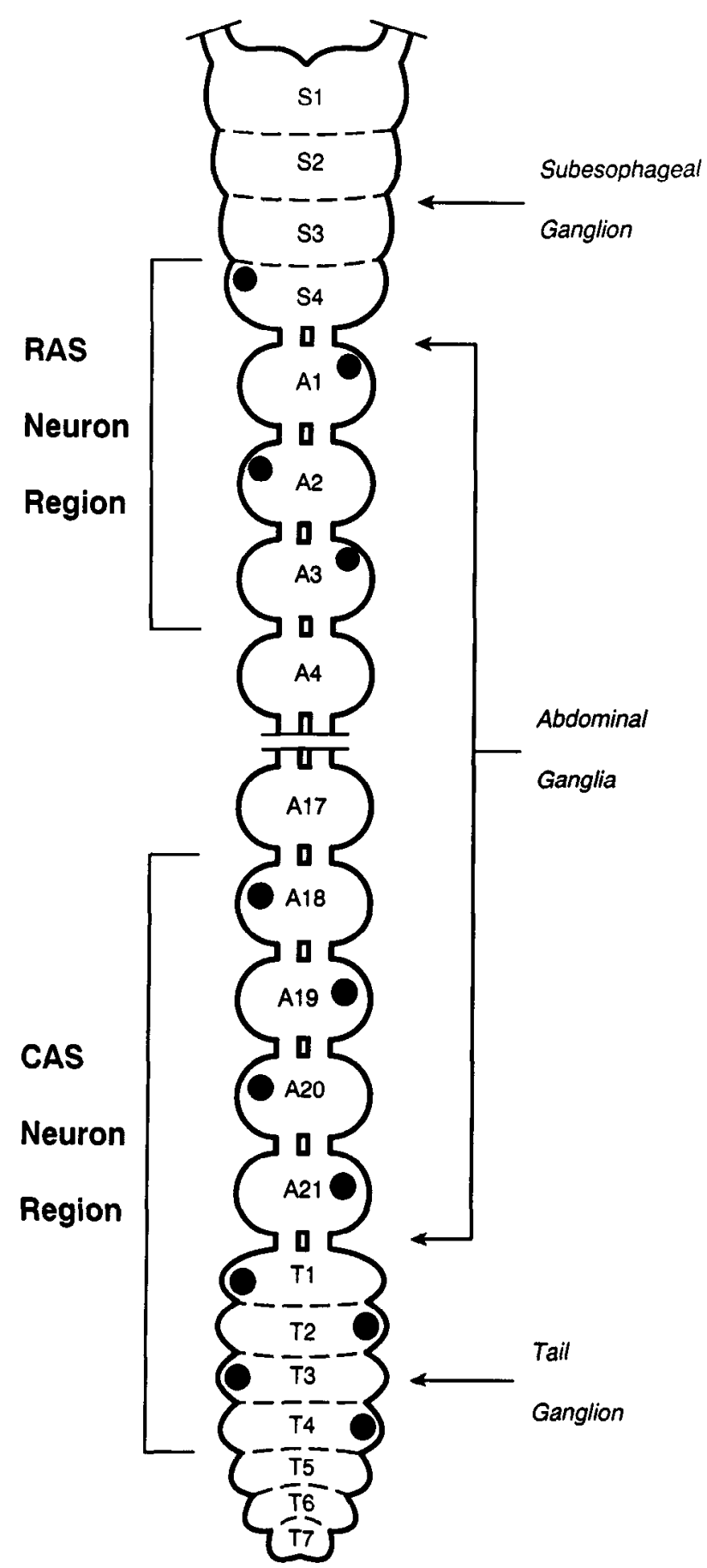

Figure 1. Schematic diagram of the distribution of RAS and CAS neurons in the leech nerve cord. The nerve cord is divided into the 4 fused segments of the subesophageal ganglion $(S I-S 4)$, the 21 abdominal ganglia $(A 1-A 21)$, and the 7 fused segments of the tail ganglion $(T 1-T 7)$. RAS is found in segments $S 4-A 3$, while CAS is found in segments A18-T4.

for most pairs of adjacent segments) in their respective domains (Fig. 1).

Previous work has shown that both AS neurons arise in the embryo from bilaterally paired sets of immature neurons (Shankland and Martindale, 1989). Within a ganglion, one neuron of each pair takes on the persistently immunoreactive AS phenotype, while its contralateral homologue expresses the same immunoreactivities transiently or not at all. Experimental studies have shown that both right and left homologues are capable of manifesting the mature AS phenotype and suggest that there is a determinative interaction between these 2 neurons that is responsible for their asymmetric differentiation (Martindale and Shankland, 1990). When a selected group of neurons, including an immature AS homologue, are ablated on one side of an embryonic nerve cord, the contralaterally homologous neuron takes on the mature AS phenotype in over $90 \%$ of the lesioned ganglia. Therefore, the choice as to which side of the ganglion will generate the mature AS neuron is not wholly predetermined at the time these neurons are born, but rather, is controlled by cell interactions. Timed ablations reveal that this interaction takes place after the neurons have undergone their terminal mitosis (Martindale and Shankland, 1990). In a formal sense, the interaction of bilateral AS homologues represents a type of neuronal competition, in that only 1 of the 2 cells will take on the mature AS phenotype (the primary developmental fate; see Kimble, 1981), while the other homologue, if allowed to develop, is diverted into some other, secondary developmental fate. It is not known whether the nonimmunoreactive homologue undergoes cell death or simply takes on a different profile of neuropeptide expression (Shankland and Martindale, 1989).

It is less clcar how the AS neurons come to alternate sides in successive ganglia. Alternation could derive from individual segments having a right- or left-handed bias that affects the outcome of the intraganglionic competition. On the other hand, the alternation might result from interactions between ganglia, such that the neuronal asymmetries established in one ganglion would influence the competition of right and left homologues in other, neighboring ganglia. Evidence of such interactions was previously obtained by transecting the embryonic nerve cord prior to the appearance of AS neuron asymmetry. Transections of the posterior nerve cord significantly reduced the probability of CAS neuron alternation in the vicinity of the lesion (Martindale and Shankland, 1990).

To obtain a better understanding of the factors that govern AS neuron differentiation, in this study, we have used cell ablations to impose an asymmetry on single developing leech ganglia and have examined the degree to which such imposed asymmetries influence development in adjacent ganglia. The cell ablation techniques utilized in this paper rely on labeling the AS neurons with a fluorescent lineage tracer by the prior injection of their teloblast ancestors and using the fluorescent dye either as a photo-oxidizing agent or as a visible marker to target cells for destruction with a laser microbeam. The unilateral ablation of immature AS neuron homologues insures the formation of an immunoreactive AS neuron on the side of the ganglion contralateral to the lesion; immunostaining of these nerve cords revealed a strong tendency for the neighboring ganglia to develop an asymmetric AS neuron on the side predicted by an imposed pattern of segmental alternation. Similar results were obtained following ablation of either RAS or CAS neuron homologues and demonstrate the presence of interganglionic cell interactions that influence the neuropeptide phenotype choice of these 2 neurons.

\section{Materials and Methods}

\section{Animals}

These experiments used embryos of 2 closely related species of the glossiphoniid leech Helobdella, which have essentially identical RAS and CAS neuron distributions (see Martindale and Shankland, 1990). 
A

\section{Step 1}

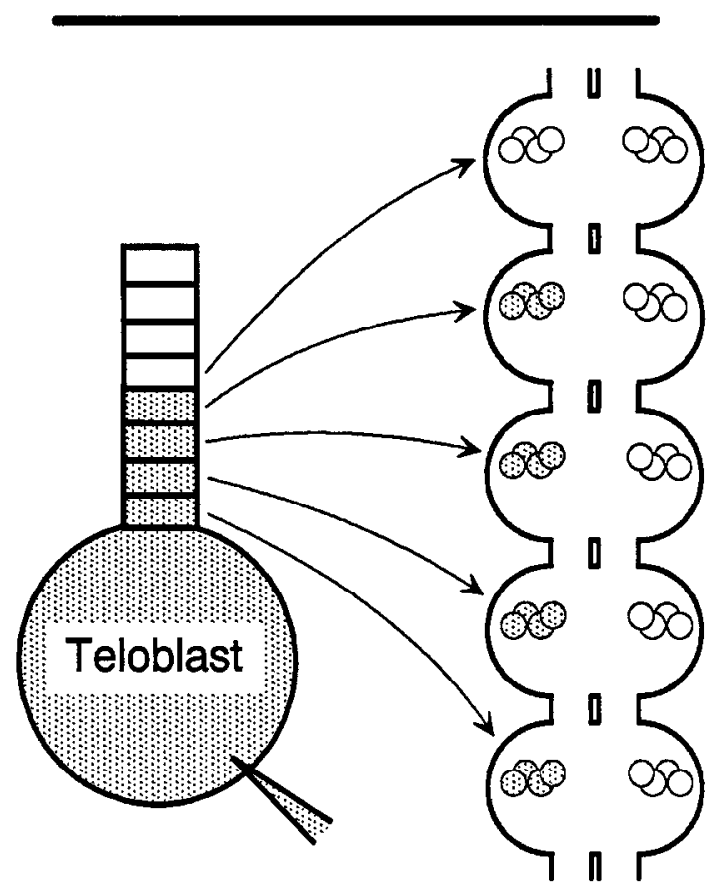

Step 2

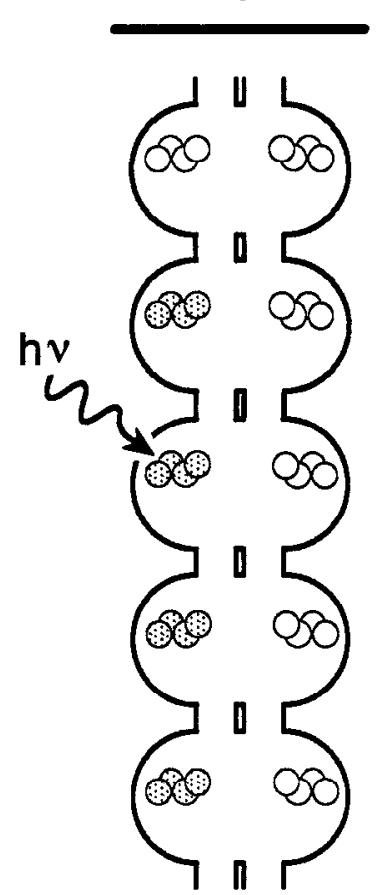

$\mathbf{B}$

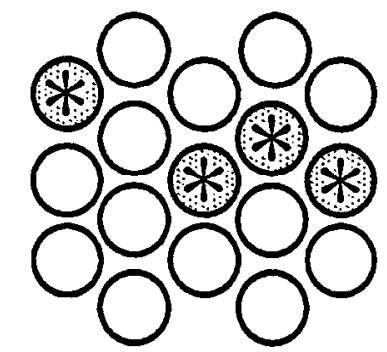

C

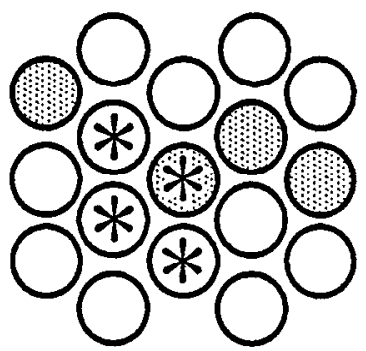

Figure 2. Methods used to ablate single AS neuron homologues. $A$, In both techniques, a subset of neurons are labeled with a fluorescent dextran (stippling) by prior injection of the ancestral teloblast. Each teloblast gives rise to a longitudinal chain of blast cells, and those blast cells produced after the injection contain the fluorescent dye. Each blast cell then gives rise to a characteristic subset of the neurons on 1 side of a specific segmental ganglion. The fluorescent label was used to target neurons AS neurons for photoablation. $B$, In one method, the labeled neurons were selectively ablated by photoexcitation of the fluorescent dye eosin within the living cells. This method destroys $\left(^{*}\right)$ only those neurons that have inherited the fluorescent dye, regardless of their spatial arrangement. $C$, In the second method, the fluorescent dye was used to target a laser microbeam. It is presumed that the laser destroys a small group of spatially contiguous cells $\left(^{*}\right)$, regardless of which cells contain the fluorescent label.

Embryonic stages are described in Stent et al. (1982). Adult and juvenile Helobdella were taken from laboratory breeding colonies, while adult leeches of another glossiphoniid species, Theromyzon rude, were captured in the wild and kindly provided to us by Duncan Stuart.

\section{Ablating AS precursors in selected hemiganglia}

Two different methods were used to ablate AS neurons or their precursors in selected hemiganglia, both of which rely on selectively labeling a subset of the neurons within the ganglion with a fluorescent tracer (Fig. 2A). The leech nerve cord arises from a number of identifiable, bilaterally paired blastomeres, termed teloblasts, and by injecting lineage tracers into a selected teloblast, it is possible to label all of its descendants (Weisblat et al., 1980). This technique was previously used to show that the RAS neurons arise from the lineally identified $\mathrm{N}$ teloblast, whereas the CAS neurons arise from a lineally distinct $M$ teloblast (Shankland and Martindale, 1989).

Dye-sensitized photoablation. If a cell lineage is labeled with a fluorescent tracer, the dye-containing cells are sensitized to light, and strong irradiation can be used to ablate either the injected cell or its descendants (Shankland, 1984). As in the previous study (Martindale and Shankland, 1990), we used this technique to ablate RAS or CAS neurons in the developing nerve cord. A teloblast ( $\mathrm{N}$ or $\mathrm{M}$ ) was injected with a combination of the highly fluorescent lineage tracer rhodamine-dextranamine (RDA; synthesized by the method of Gimlich and Braun, 1985) and the photosensitizer eosin-dextran-amine (EDA; Molecular Probes, Eugene, OR). The injected embryo was then reared to late stage 9 or early stage 10, at which time the nerve cord is formed, but the side of AS development is not yet determined. The embryo was relaxed and mounted ventral side up under a coverslip, and the dye-containing teloblast progeny were illuminated at $485 \mathrm{~nm}$ using a fluorescence microscope, leading to the selective destruction of the EDA-containing cells. In the present study, the illumination was limited to the AS homologue-containing regions of selected single hemiganglia. After developing to late stage 11, the embryos were fixed, and the nerve cords were stained with anti-SCP as previously described (Shankland and Martindale, 1989). The loss of cells labeled with RDA/EDA indicated the site of the ablation, and the pattern of AS neuron development could be ascertained by the distribution of immunoreactivity.

This technique has the advantage that only those ncurons containing tracer dye (those derived from the same teloblast) are ablated within a selected hemiganglion (Fig. $2 B$ ). This number can be quite large when ablating RAS homologues, because the $\mathrm{N}$ teloblast makes approximately 100 neurons per hemiganglion, but is quite small when ablating CAS, because the $M$ teloblast makes only 5 neurons per hemiganglion (Kramer and Weisblat, 1985; Shankland and Martindale, 1989). There is, however, the possibility with this technique that scattered light will sublethally damage dye-containing cells in adjacent ganglia.

Laser ablation. A dye-pulsed laser (Laser Sciences VSL-337, using coumarin 440) was used to ablate cells in the immature nerve cord. The beam was focused with a $10 \times$ objective, directed into the epi-illuminator path of a Zeiss Standard microscope with 2 mirrors, and refocussed into the image plane of the microscope objective (Zeiss $40 \times$ tripleimmersion Neofluar). The filter holder was mounted with a Zeiss FT 510 dichroic mirror to reflect the beam into the back of the objective. The epi-illuminator port was equipped with a 2-way mirror housing so that it was possible to switch between the laser and a mercury arc, allowing us to aim the laser at particular fluorescently labeled cells.

The experimental procedure was similar to that used for photoablation, except that only RDA was injected. Because of the small diameter of the laser beam ( $<5 \mu \mathrm{m}$ on the specimen), damage should be limited to a small number of cells in the target hemiganglia, with little or no damage to adjacent ganglia. However, it is expected that cell bodies immediately surrounding the targeted dye-containing neurons (but not 


\section{RAS Neuron}

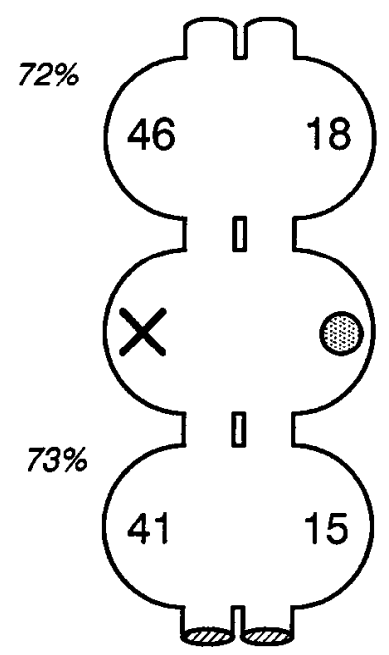

\section{CAS Neuron}

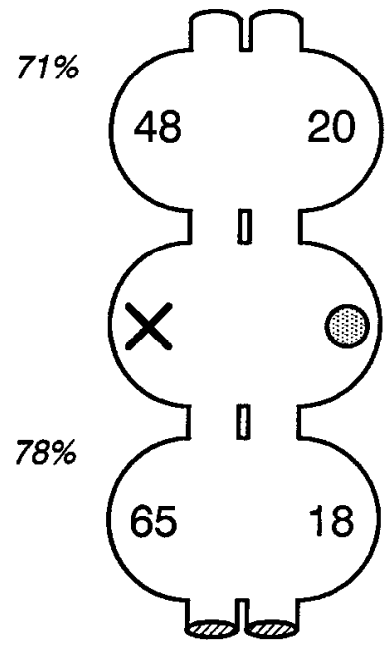

Figure 3. Effect of unilateral RAS and CAS neuron ablations on asymmetry of AS homologue neuron differentiation in adjacent segments. Ablation of a single AS neuron homologue $(X)$ causes the contralateral homologue to become a mature, immunoreactive AS neuron (stippled circle). Numbers represent the total of experimental embryos in which an unpaired, immunoreactive AS neuron was observed on the side ipsilateral or contralateral to the lesion in each of the 2 adjacent ganglia. There was a strong tendency for AS neurons in the adjacent ganglia to alternate sides with the AS neuron in the lesioned ganglia, with the percentage of alternation shown in italics.

necessarily containing the fluorescent tracer) would be ablated, as well (Fig. 2C). Because of the large number of neurons labeled by the injection of an $N$ teloblast, it would be difficult to identify and reliably eliminate the RAS neuron or its precursor with the focused laser beam. Hence, this technique was used exclusively to perform ablations in the CAS region.

\section{Lucifer fills}

CAS neurons were injected with Lucifer yellow $\mathrm{CH}$ (Sigma) to determine the extent of their adult branching pattern. Animals were dissected in leech paralysis solution (Martindale and Shankland, 1990), and their nerve cords were removed with forceps. Nerve cords from the midbody through the tail ganglion were pinned ventral side up with small wickets fashioned from 0.001-inch tungsten wire (California Fine Wire Co.; Grover City, CA) on a Sylgard-coated microscope slide in a modified leech Ringer's solution [115 mM NaCl, $4 \mathrm{~mm} \mathrm{KCl}, 1.8 \mathrm{~mm} \mathrm{CaCl}_{2}, 10$ mM HEPES (pH 7.4)] to which $10 \mathrm{~mm}$ glucose was added. Nerve cords were treated for 3-5 min at room temperature with $0.25 \%$ collagenase (Sigma, type I) in this same solution to soften the ganglion capsule for microelectrode penetration, then washed. Neuronal cell bodies were visualized with Nomarski optics, and potential CAS neurons were impaled with 40-80 M $\Omega$ glass microelectrodes filled with $5 \%$ Lucifer yellow, which was iontophoresed into the cell with $0.5-2.0-n A$ hyperpolarizing current. CAS neuron resting potentials were measured at $15-25 \mathrm{mV}$, and action potentials could not be elicited. Injected nerve cords were depinned and washed in Ringer's solution at $4^{\circ} \mathrm{C}$ for $30-90 \mathrm{~min}$ so the dye would spread to distant parts of the cell, then pinned again and fixed overnight with $4 \%$ formaldehyde in HEPES buffer (pH, 7.4). Lucifer yellow-injected nerve cords were then stained with anti-SCP as previously described (Shankland and Martindale, 1989), using a rhodamine-conjugated secondary antibody.

\section{Statistics}

Comparisons of data to a 50-50 binomial distribution (single-tailed test) were performed using Statpak software.
Table 1. Distribution of mature AS neurons in embryos subjected to the lesion of single AS neuron homologues

\begin{tabular}{|c|c|c|c|c|c|c|c|c|c|}
\hline $\begin{array}{l}\text { RAS } \\
\text { region }\end{array}$ & $\mathrm{C}$ & $\mathrm{P}$ & I & 0 & $\begin{array}{l}\text { CAS } \\
\text { region }\end{array}$ & $\mathrm{C}$ & $\mathbf{P}$ & I & 0 \\
\hline$S 4^{a}$ & - & - & - & - & $\mathrm{A} 18^{a}$ & - & - & - & - \\
\hline Al & 1 & 0 & 13 & 0 & A19 & 4 & 5 & 31 & 0 \\
\hline $\mathrm{A} 2$ & 12 & 1 & 2 & 0 & A20 & 19 & 5 & 10 & 1 \\
\hline A3 & 4 & 0 & 10 & 0 & A21 & 12 & 3 & 14 & 1 \\
\hline S4 & 5 & 1 & 15 & 0 & A18 & 11 & 1 & 25 & 0 \\
\hline $\mathrm{Al}^{a}$ & - & - & - & - & $\mathrm{A} 19^{a}$ & - & - & - & - \\
\hline $\mathrm{A} 2$ & 7 & 1 & 14 & 0 & A20 & 11 & 1 & 17 & 1 \\
\hline A3 & 14 & 0 & 6 & 1 & A21 & 13 & 0 & 11 & 3 \\
\hline S4 & 14 & 0 & 5 & 0 & A18 & 14 & 0 & 8 & 0 \\
\hline A1 & 6 & 1 & 13 & 0 & A19 & 8 & 0 & 14 & 0 \\
\hline $\mathbf{A} 2^{a}$ & - & - & - & - & $\mathrm{A} 20^{a}$ & - & - & - & - \\
\hline A3 & 7 & 0 & 14 & 0 & A21 & 3 & 0 & 17 & 2 \\
\hline S4 & 12 & 0 & 12 & 0 & A18 & 5 & 0 & 5 & 0 \\
\hline Al & 14 & 0 & 10 & 0 & Al9 & 5 & 0 & 5 & 0 \\
\hline $\mathrm{A} 2$ & 7 & 0 & 17 & 0 & A20 & 1 & 0 & 9 & 0 \\
\hline $\mathrm{A} 3^{\alpha}$ & - & - & - & - & $\mathrm{A} 21^{a}$ & - & - & - & - \\
\hline
\end{tabular}

This table shows the number of embryos in which a ganglion contained unpaired AS neurons contralateral to the lesion (C), paired AS neurons (P), unpaired AS neurons ipsilateral to the lesion (I), or no detectable AS neuron (0). Cases were included only if an AS neuron appeared in the same ganglion contralateral to the lesion. For each ncrve cord scorcd, individual ganglia that were damaged or lost during the dissection were not included, resulting in discrepancies between the numbers of ganglia scored in each category of ablation. Data in the table are grouped by experiment.

a Segment lesioned.

\section{Results}

Single AS neuron homologues were ablated by either of 2 photoablation techniques (Fig. 2), and the pattern of neuronal differentiation was scored by staining the mature AS neurons with anti-SCP. The 2 techniques yielded similar results, as did ablations on the right and left sides of the nerve cord. Following ablation of a single RAS neuron homologue, an immunoreactive RAS neuron was observed to develop on the side contralateral to the lesion in 82 of 89 cases (92\%). Ablation of a single CAS neuron homologue resulted in an immunoreactive CAS neuron developing contralateral to the lesion in 109 of 121 cases $(90 \%)$. In most instances, this immunoreactive AS neuron displayed the cell body position and axonal branching pattern typical of homologues in unlesioned ganglia. Therefore, ablation of single AS neuron homologues is sufficient to force the contralateral homologue to take on the immunoreactive AS fate, including the majority of cases in which that cell would not otherwise have done so.

\section{Effect of ablations on immediately adjacent ganglia}

Ablation of a single AS neuron homologue produced a signifcant bias in the side of AS development in adjacent ganglia (Table 1). In the RAS region of the nerve cord, the dye-sensitized photoablation of a single RAS neuron homologue and related neurons clearly biased the side of RAS neuron development in both immediately anterior and posterior ganglia (Fig. 3), with the majority of the RAS neurons in adjacent ganglia developing on the side contralateral to the RAS neuron in the lesioned ganglion (Fig. 4). Of 119 cases in which an adjacent ganglion contained an asymmetrically immunoreactive RAS neuron, this neuron was located on the same side as the lesion, that is, al- 


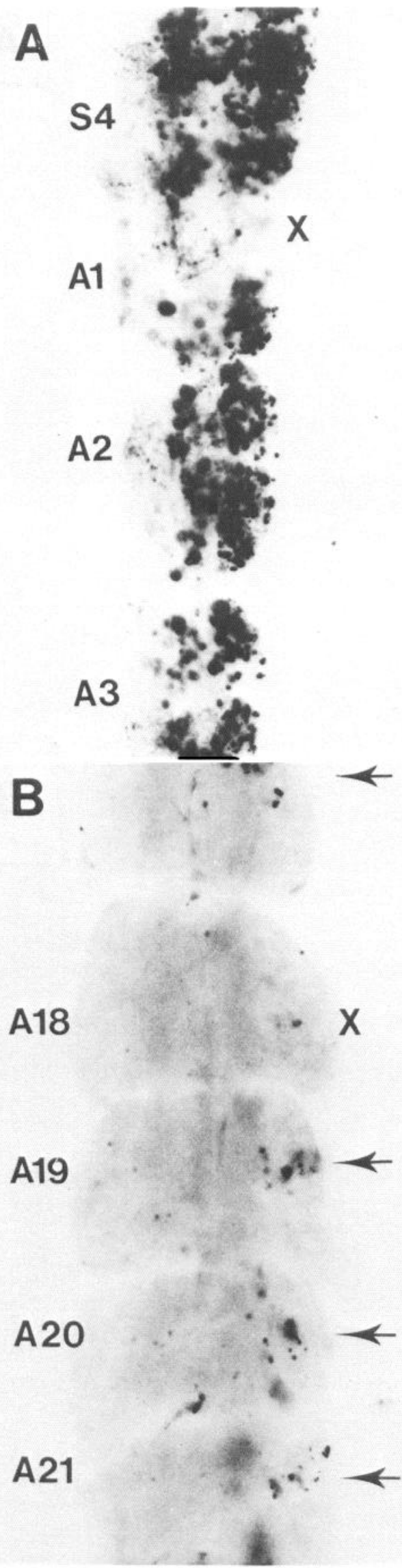

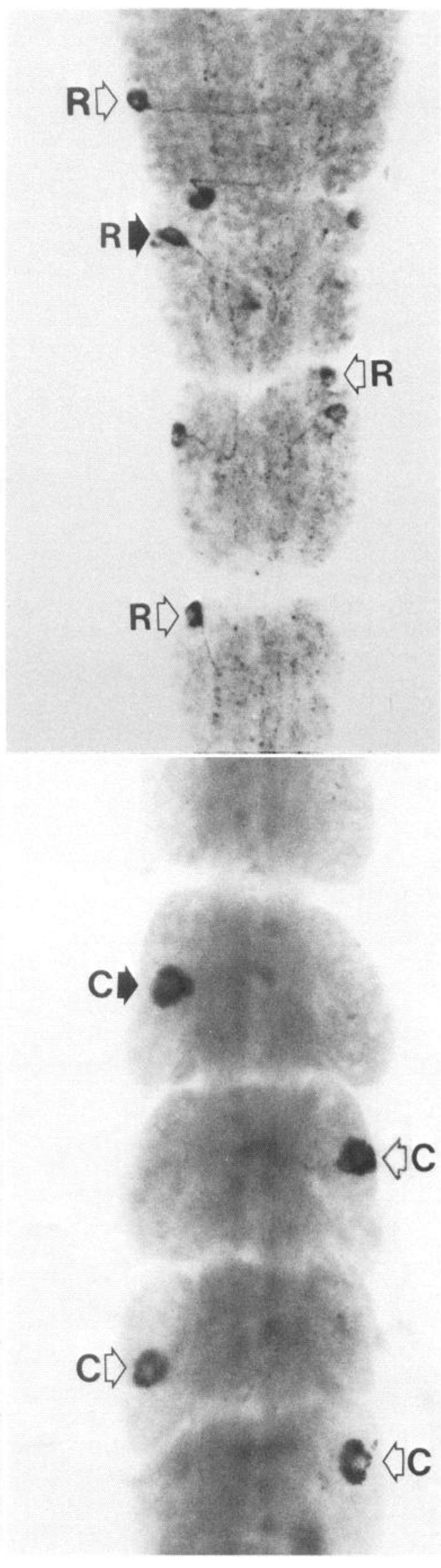

Figure 4. Nerve cords from embryos in which single AS homologue neuron was ablated. These photomicrographs are fluorescence negatives, oriented with the anterior to the top of the page. Each part consists of 2 photographs taken from a single nerve cord. The left image presents the rhodamine fluorescence resulting from the distribution of the lineage tracer RDA within the nerve cord, and the right image presents the distribution of fluorescein fluorescence resulting from anti-SCP staining. $A$, RAS region of a photoablated embryo. The lesion $(X)$ can be seen by the paucity of RDA-labeled (i.e., $\mathrm{N}$ teloblast-derived) cells on the right side of the nerve cord in segment $A 1$. An immunoreactive RAS neuron ( $R$, solid arrow) is observed in the contralateral, unablated hemiganglion. This RAS neuron alternates sides with the RAS neurons $(R$, open arrows) in more posterior ganglia, but lies on the same side as the RAS neuron in $S 4$. B, CAS region of a laserablated embryo. RDA is present in a small cluster of $\mathbf{M}$ teloblast-derived neurons on the right side of each hemiganglion (small arrows) but is absent in ganglion $A 18$ as a result of the laser ablation $(X)$. An immunoreactive CAS neuron ( $C$, solid arrow) can be seen on the contralateral side of this ganglion and alternates sides with the CAS neurons $(C$, open arrows) in adjacent ganglia. 
ternated with the RAS neuron whose cell body occupied the lesioned ganglion, in 86 cases (72\%), a distribution that differs significantly from the expectations of a 50-50 binomial distribution $\left(p^{50-50}<10^{-3}\right.$, single-tailed test). There was also a minority of 3 cases in which an adjacent ganglion contained a pair of immunoreactive RAS neurons at the age of examination. The tendency towards alternation continued into more distant ganglia (Table 1); however, we were not able in these experiments to determine whether the lesion influenced the development of RAS neurons in nonadjacent ganglia directly, or indirectly by means of its influence on neurons in intervening ganglia.

In the CAS region of the nerve cord, both dye-sensitized photoablation and laser irradiation were used to unilaterally ablate single CAS neuron homologues. As in the RAS region, forcing CAS to develop on 1 side of a particular ganglion had a highly significant effect upon the side of CAS development in the immediately adjacent ganglia (Table 1, Figs. 3, 4). Of 151 cases in which an adjacent ganglion contained an asymmetrically immunoreactive CAS neuron, this neuron alternated with the CAS neuron in the lesioned ganglion in 113 cases (75\%), a distribution that differs significantly from the expectations of a binomial $\left(p^{50-50}<10^{-3}\right)$. There was also a minority of 7 cases in which an adjacent ganglion contained a pair of immunoreactive CAS neurons and 3 cases in which an adjacent ganglion contained no visibly immunoreactive CAS neuron.

The RAS and CAS neurons differ in their anterior and posterior axonal projections (Shankland and Martindale, 1989), and one might have expected a corresponding difference in the degree to which they influence development of homologues in anterior and posterior ganglia. In fact, single ganglion ablations of either neuron have a similar effect on the development of both the next anterior and the next posterior ganglion (Fig. 3). When the total anterior- and posterior-going effects for RAS and CAS regions were statistically compared, no significant difference was observed $\left(2 \times 4 \chi^{2}\right.$ test, $\left.p^{0}>0.1\right)$.

The RAS neuron shows little or no segmental variation of alternation frequency in normal animals, and we did not observe significant segmental variation in the strength of interganglionic interactions $\left(2 \times 3 \chi^{2}\right.$ test, $\left.p^{0}>0.1\right)$. We did, however, observe significant variation in the apparent strength of interaction between certain pairs of segmental ganglia within the CAS region of the nerve cord $\left(2 \times 3 \chi^{2}\right.$ test, $\left.0.05>p^{0}>0.025\right)$. When both anterior- and postcrior-going cffects were summed, the cxpcrimentally induced frequencies of alternation between ganglia $\mathrm{A} 18$ and A $19(78 \%)$ and between ganglia A20 and A21 (87\%) differed significantly from that expected from a 50-50 binomial distribution $\left(\mathrm{A} 18 / 19, p^{50-50}=5 \times 10^{-7} ; \mathrm{A} 20 / 21, p^{50-50}=3 \times 10^{-5}\right)$. In contrast, the experimentally induced frequency of alternation between $\mathrm{A} 19$ and $\mathrm{A} 20$ was smaller $(62 \%)$ and only marginally significant $\left(p^{50-50}=0.06\right)$. This segmental difference in the apparent strength of interganglionic interaction correlates with the relatively low frequency of alternation observed between ganglia A19 and A20 in normal animals (Shankland and Martindale, 1989). The fact that there are parallel changes in both normally occurring and experimentally induced alternation frequency for certain pairs of segmental ganglia further supports the idea that the cell interactions revealed by these experiments are indeed responsible for the normal establishment of segmental alternation.

\section{Ablations in segments not containing $A S$ neurons}

Although mature RAS and CAS neurons are restricted to precisely delimited segmental domains, apparent homologues of these cells are born and transiently express SCP- and FMRFamide-like immunoreactivities in other segments of the embryo (Shankland and Martindale, 1989). Can transiently immunorcactive homologues influence the asymmetric differentiation of true CAS neurons located in adjacent segments?

To address this question, we unilaterally ablated a single CAS neuron homologue in ganglion A17, 1 segment anterior to the front border of the adult CAS neuron domain. The CAS neuron is 1 of 4 laterally situated neurons derived from the $M$ teloblast in each hemiganglion (Shankland and Martindale, 1989), and in a series of 18 embryos, all 4 of these neurons were unilaterally destroyed in segment A17. In 12 cases, an immunoreactive CAS neuron developed in A18 on the side contralateral to the lesion, and in 6 cases, on the side ipsilateral to the lesion. This distribution was not significantly different from that of the binomial $\left(p^{50-50}=0.12\right)$. On the other hand, this distribution was dramatically different from the strong posterior-going tendency towards alternation observed when an ablation was performed in one of the ganglia that does form an immunoreactive CAS neuron $\left(2 \times 2 \chi^{2}\right.$ test, $\left.p^{0}<10^{-3}\right)$. Thus, the CAS ncuron homologucs in ganglion A18 would seem to have little or no developmental interaction with their homologues in ganglion $\mathrm{A} 17$ and are not influenced by those homologues in the same way that CAS neurons in other ganglia are influenced by more anterior CAS neurons.

\section{Axonal projections of the CAS neuron}

Antibody staining has revealed that both RAS and CAS neurons project axons into neighboring ganglia (Shankland and Martindale, 1989). Such projections could play a role in the interganglionic interactions described above, and we therefore undertook a more detailed analysis of these axonal morphologies by injecting CAS neurons with the fluorescent dye Lucifer yellow (Fig. 5). CAS neurons located in ganglia A18-A20 were injected in a total of 16 postembryonic Helobdella, as well as 5 postembryonic leeches of the species Theromyzon rude. Every CAS neuron possesses a single large-caliber axon, henceforth termed the primary axon, which crosses the midline of the neuropil, turns to enter a medial fiber tract within the anterior contralateral connective, and projects as many as 4 segments anteriorly. Occasionally, a second Lucifer yellow-filled process of finer caliber could be seen to fasciculate with or run parallel to the primary axon within the connective. In the ganglion of origin, the CAS neuron arborizes on both sides of the neuropil (Fig. $5 A$ ), but in more anterior ganglia, the primary axon extends only a few branches, which rarely cross the midline (Fig. $5 D, E$ ).

In addition to the large-caliber primary axon, Lucifer yellowfilled CAS neurons displayed a variable array of finer caliber, secondary axons that extended through 1 or more of the other connectives (Fig. $5 A, B$ ). In most cases in which secondary axons were observed, they also traveled in the extreme medial region of the connective occupied by the primary axon of other CAS neurons (Fig. 5C). Secondary axon variation was observed for even the most intensely labeled cells, suggesting that their presence or absence represents morphological variability and not the uncertainty of visualizing fine-caliber processes. Secondary axons were observed in both species, and Figure 6 summarizes for Helobdella the frequency with which interganglionic axons were observed in each of the lateral connectives.

The interganglionic projections of the CAS neuron probably mediate the cellular interactions described in this paper, and it is of interest to evaluate the cell's structure in this light. For example, the CAS neurons in ganglia A19 and A20 normally 


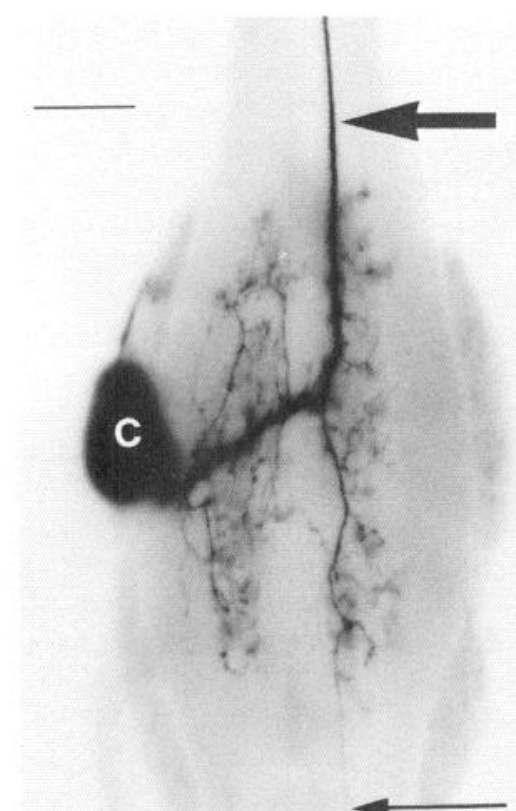

a

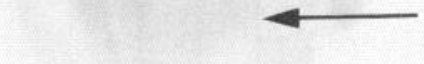

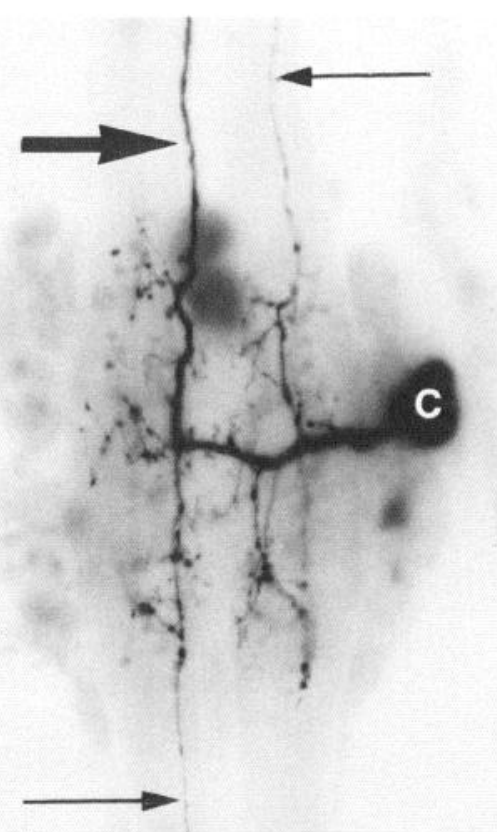

b

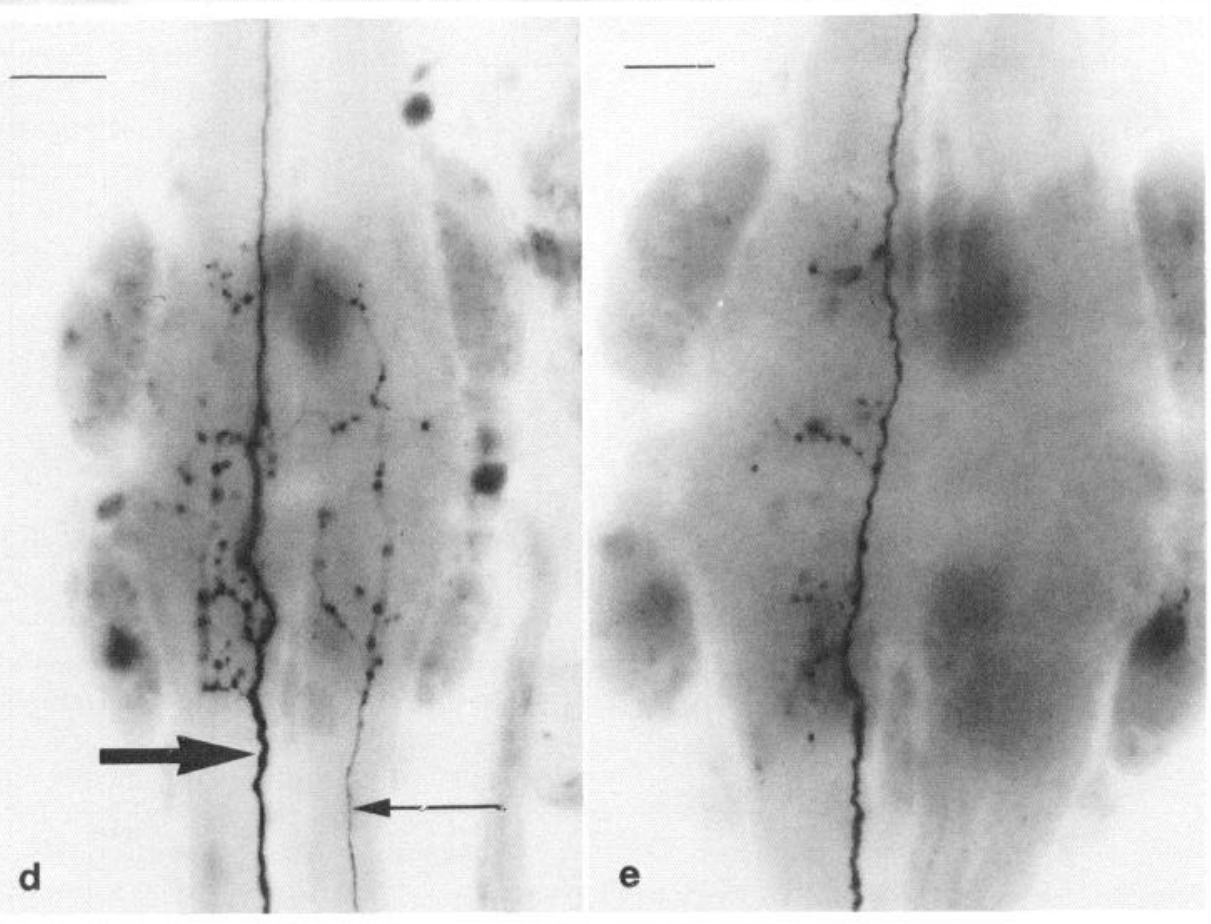

Figure 5. Branching pattern of CAS neurons in adult leeches as revealed by Lucifer yellow injection $(a, b, d, e)$ and anti-SCP staining $(c)$. These photomicrographs are fluorescence negatives, with the anterior oriented towards the top of the page. $a$, CAS neuron in ganglion A20 of the species Theromyzon rude. Note the large primary axon (large arrow) projecting anteriorly within the contralateral connective. This cell also possesses a fine secondary axon (small arrow) projecting into the posterior contralateral connective. $b-e$, CAS neuron with cell body $(C)$ located in ganglion A 18 of the leech Helobdella. In $b$, Lucifer yellow injection reveals a large primary axon (large arrow), as well as 2 secondary axons (small arrows). In $c$, anti-SCP staining of the same ganglion demonstrates that the injected cell is the asymmetrically immunoreactive CAS neuron. Note that the axons of the injected cell (small arrows) can be seen by immunoreactivity alone, as can the primary axon of the CAS neuron in the next posterior ganglion (large arrow). $d$ shows the Lucifer yellow-filled primary (large arrow) and secondary (small arrow) axons of the injected neuron in the next anterior ganglion, and $e$ shows the primary axon projecting into the second ganglion anterior to the cell body. Note that the interganglionic axons branch almost exclusively to 1 side of the ganglion midline. Scale bars: $a-c, 35 \mathrm{~mm} ; d, 30 \mathrm{~mm} ; e, 25 \mathrm{~mm}$.

show a much lower frequency of alternation (approximately $80 \%$ ) than that seen between ganglia A18 and A19 (approximately $100 \%$ ), suggesting that the interaction is less robust (Shankland and Martindale, 1989). Four of the CAS neurons examined by Lucifer yellow injection had cell bodies located in ganglion A20: in 2 cases, the injected CAS neuron was located on the same side as, and in 2 cases, on the side opposite to, the immunoreactive CAS neuron in the next anterior ganglion. However, all 4 of these A20 CAS neurons displayed the same general morphology, with a primary axon projecting into the 


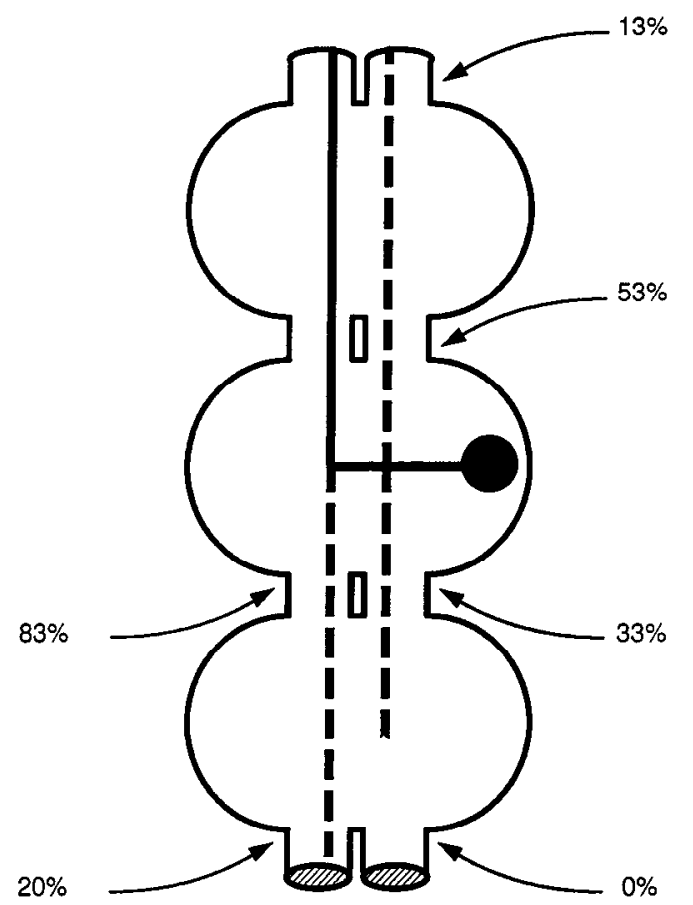

Figure 6. Variability in interganglionic projections of mature CAS neuron. Every CAS neuron has a primary axon (solid line) that projects into more anterior ganglia through the contralateral connective. In addition, CAS neurons displayed secondary interganglionic axons (dashed lines) that were variable in their number and array. Data are taken from 15 CAS neurons that were filled with Lucifer yellow in adult Helobdella, and percentages reflect the number of instances in which a Lucifer yellow-filled secondary axon was observed in a given connective nerve.

anterior contralateral connective. Moreover, the CAS neurons in all 3 segments exhibited a similar, albeit variable, array of secondary axons. Taken together, these findings argue that neither the differences between segments nor the occasional failures of alternation result from gross differences in the axonal projection of the CAS neurons. Rather, it seems likely that the less robust interaction between neurons in certain ganglion pairs involves differences in the timing of axonal outgrowth or in cell recognition.

\section{Discussion}

The unpaired AS ncurons of the adult leceh nerve cord have a spatially coordinated distribution in which there is a pronounced tendency for cells to alternate from right to left sides in successive segmental ganglia. Each AS neuron arises from a bilateral pair of immature homologues by a process of asymmetric differentiation (Shankland and Martindale, 1989). In most cases, both right and left homologues begin to express SCP- and FMRFamide-like immunoreactivities, but only 1 continues to express these immunoreactivities into postembryonic life and becomes the mature AS neuron. Previous ablation studies showed that homologues on both sides of the ganglion are competent to differentiate as mature AS neurons, because if selected neurons (including the postmitotic AS homologue) are ablated on one side, the contralateral AS homologue nearly always acquires the mature AS phenotype (Martindale and Shankland, 1990). Those findings indicated that there is an intraganglionic inter- action in which neurons on the 2 sides compete in some way for the AS fate. The results of the present study indicate that there is also an interaction between ganglia and suggest that this interaction may account for the normally observed pattern of segmental alternation. If an AS neuron is forced to appear on one side of a ganglion by ablation of its contralateral homologue, the mature AS neurons in immediately adjacent ganglia are not randomly distributed, but rather, show a pronounced tendency to lie on the opposite side. Therefore, the choice of an immature AS neuron homologue to maintain or lose specific neuropeptide immunoreactivities is not predetermined by that cell's lineage history, but rather, is controlled by cell interactions both within and between ganglia.

It seems likely that the signal that biases AS neuron asymmetry in neighboring segments originates from the surviving AS neuron in the lesioned ganglion. The immunoreactive AS neurons in immediately adjacent ganglia were more likely to be located on the same side as (i.e., closer to) the site of the lesion, arguing against any significant role for nonspecific damage. Similarly, the results of this study cannot be accounted for by sublethal toxicity of the intracellular lineage tracers, because the AS neurons in adjacent ganglia tended to be located on the side of the nerve cord that was labeled. There are numerous examples in which a cell's choice between alternative developmental pathways involves interaction with specific, lineally homologous cells (Sulston and White, 1980; Kimble, 1981; Weisblat and Blair, 1984; Stent, 1985), and in the following discussion, we have envisioned the AS neuron surviving opposite the lesion as the primary source of the information that affects the developmental asymmetry of its homologues in other segments. However, we cannot rule out the possibility that the observed effects result from destruction of some other cell(s) closely related to, and in close physical proximity to, each of the 2 AS neurons.

\section{Can the demonstrated interganglionic interactions account for normal alternation?}

Unilateral lesions of single AS neuron homologues strongly bias the distribution of mature AS neurons in adjacent ganglia. The mature AS homologue that forms in the lesioned ganglion exhibits a normal pattern of immunoreactivity and branching, suggesting that its interaction with homologues in neighboring segments is characteristic of the interactions that occur during normal development. However, the experimentally induced pattern of alternation is not as reliable as that seen in unperturbed embryos. The AS neurons normally alternate sides with a frequency ranging from $80 \%$ to virtually $100 \%$ for specific ganglion pairs (Shankland and Martindale, 1989), but the cumulative alternation frequency generated by experimentally induced asymmetries was only $73 \%$.

Several factors could account for this discrepancy. One possibility is that AS homologues in the adjacent ganglia may already have had a right- or left-handed bias at the time of the lesion. Even if we assume that the asymmetry of AS differentiation is determined entirely by postmitotic cell interactions, it is probable that the interaction between ganglia has already begun when our ablations are performed at the beginning of stage 10. The CAS neurons of Helobdella extend their interganglionic axons $2 \mathrm{~d}$ prior to this time (Shankland and Martindale, 1989). Moreover, the previous study included a day-by-day analysis of the AS neuron response to the removal of intraganglionic interactions, and it appears that some AS homologues 
already manifest an irreversible commitment to the nonimmunoreactive fate early in stage 10 (Martindale and Shankland, 1990). If a differentiating AS neuron has already biased AS development in the adjacent segments at the time of its ablation, interactions occurring after the ablation will not always be able to counteract the earlier effect. Indeed, those normally occurring cases in which AS neurons in adjacent segments fail to alternate could reflect instances in which randomly oriented intraganglionic interactions have had an effect before the interganglionic influence can hold sway.

A second possible cause for the relatively low frequency of experimentally induced alternation is that the underlying cell interactions may not be as robust as those that occur during normal development; that is, the ablation may have nonspecifically impaired the ability of the surviving, contralateral AS neuron homologue to interact with adjacent segments. This would be especially true if we imagine the interaction depending on axonal communication, because the main axon of the unablated AS homologue passes through the lesioned hemiganglion and could be receiving sublethal damage or responding to the destruction of surrounding tissues.

Another possibility is that the nonimmunoreactive contralateral homologue of the AS neuron could also play an active role in eliciting alternation. In this scenario, the asymmetric differentiation of AS neurons would be biased by the cumulative influences of their immunoreactive and nonimmunoreactive homologues. Thus, the strength of the interganglionic bias towards asymmetry produced by an immunoreactive AS neuron opposite a lesion would be less than that normally produced by an immunoreactive AS neuron and its nonimmunoreactive contralateral homologue.

\section{Cell interactions responsible for AS neuron patterning}

Several lines of evidence lead us to envision the initial event in the spatial patterning of AS neuron differentiation as the intraganglionic interaction of right and left homologues (Fig. 7A). First, this interaction is almost certainly stronger than that between segments, based on both the measured effect of cell ablations and the lack of any apparent failures of this interaction during normal development (Martindale and Shankland, 1990). Second, the axon of an AS neuron should come into proximity with that of its contralateral homologue well before it encounters the axons of its homologues whose cell bodies are located in other ganglia. Finally, the interaction between ganglia transmits information regarding AS neuron asymmetry, and there is no evidence that significant asymmetries exist prior to the intraganglionic interaction.

The interaction between bilateral homologues is competitive in nature (Martindale and Shankland, 1990). One AS homologue must inhibit acquisition of the AS phenotype in its contralateral homologue (Fig. 7A), because its ablation allows the contralateral cell to take on the AS phenotype in cases in which it would not normally do so. We imagine that right and left homologues are initially equivalent and undergo a symmetric interaction, and that 1 homologue gains an advantage and develops a strong inhibitory influence, with the result that the contralateral homologue either fails to develop or ceases to express the symmetric inhibition. Indeed, the cell that takes on the non-AS phenotype might even develop a positive feedback onto the nascent AS neuron (Fig. 7A).

The interganglionic interactions examined in this paper ap-
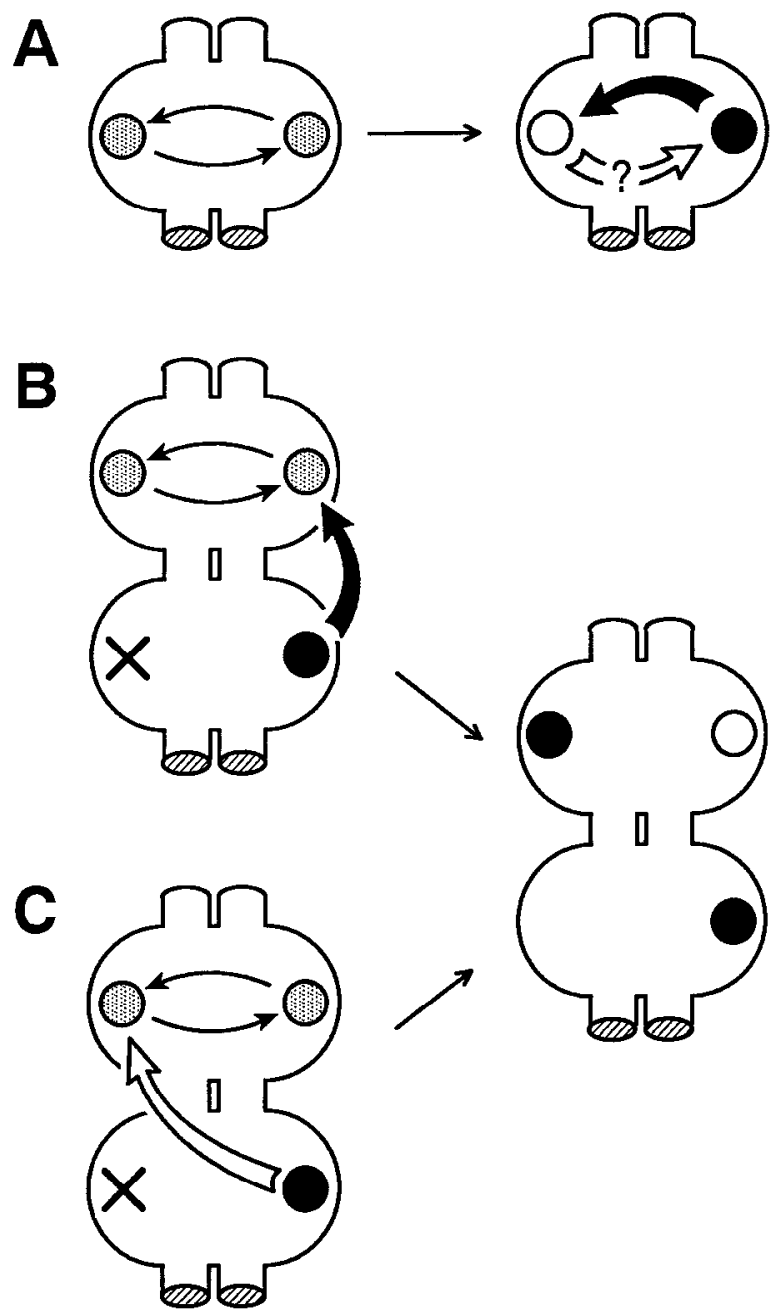

Figure 7. Schematic diagrams of postulated interactions between AS neuron homologues. Uncommitted AS homologues are represented as stippled circles; homologues committed to the AS phenotype, as solid circles; and homologues committed to the non-AS phenotype, as open circles. Cell interactions that inhibit commitment to the AS phenotype are shown as solid arrows, and cell interactions that stimulate commitment to the AS phenotype are shown as open arrows. Unspecified cell interactions are shown as thin arrows. A, Bilateral homologues within the same ganglion compete for the mature AS phenotype. It is believed that the 2 uncommitted cells undergo an initially symmetric interaction, but that 1 cell gains an advantage and begins to exert a strong inhibitory influence. This cell takes on the mature AS phenotype and forces its contralateral homologue into the non-AS phenotype. The latter cell may or may not have a stimulatory influence on the nascent AS neuron. $B$ and $C$, When an AS homologue is unilaterally ablated within a single ganglion $(X)$, the surviving contralateral homologue reliably takes on the mature AS phenotype and influences the development of paired AS homologues in the adjacent ganglia to establish a pattern of right-left alternation. Only anterior-going influences are shown here, though the experimental results reveal an equally strong influence on the next posterior ganglion. The interganglionic influence could result from inhibition of the AS phenotype in homologues on the ipsilateral side of adjacent ganglia (shown in $B$ ) and/or from the stimulation of the AS phenotype in homologues on the contralateral side of adjacent ganglia (shown in C). The present experiments cannot distinguish between these 2 interactions, because the paired homologues in the adjacent ganglion will also be interacting competitively with one another.

parently serve to bias the orientation of the intraganglionic competition. It is clear that a nascent AS neuron is, without its contralateral homologue, sufficient to bias development in the neighboring ganglia and hence must interact differently with 
Figure 8. Anterior-going primary axons (shaded) of CAS homologues from 1 segmental ganglion come into proximity with distinct ipsilateral and contralateral arborizations of CAS homologue (unshaded) located in next anterior ganglion. It is proposed that these 2 axons transmit information to that CAS homologue regarding the asymmetry of CAS neuron differentiation in the posterior ganglion and thereby influence its commitment to a CAS or non-CAS developmental pathway. Moreover, these axons would transmit information to their cell bodies of origin regarding the asymmetry of CAS neuron differentiation in the next anterior ganglion.

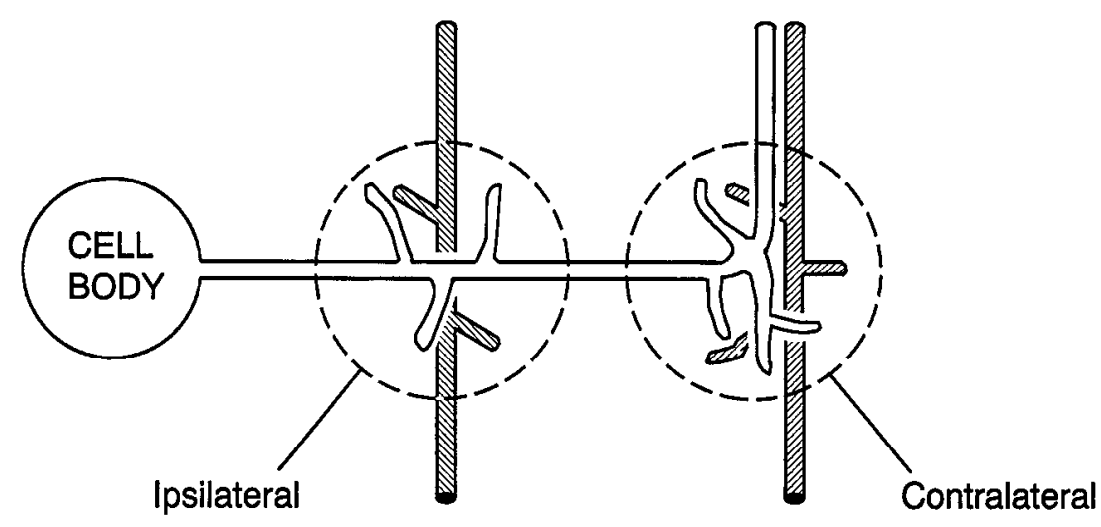

ipsilateral and contralateral homologues in those ganglia. One simple model is the assumption that all of the necessary cell interactions are mechanistically similar, and that the nascent AS neuron exerts an inhibitory interganglionic influence comparable to that occurring within a ganglion. In this model, the normal pattern of segmental alternation would arise if the strongest interganglionic influence was an inhibition between ipsilateral homologues (Fig. 7B). However, an equally feasible and nonexclusive possibility would be that the nascent AS neuron directly stimulates the AS phenotype in its contralateral homologues in the adjacent segments (Fig. 7C). In any case, it should be pointed out that the inhibitory interaction between ipsilateral homologues in adjacent ganglia cannot be stronger than any intrinsic tendency for those cells to take on the AS fate. When AS neuron homologues are ablated on one side in a string of adjacent segments, virtually all of the contralateral homologues become mature AS neurons, even though they lie on the same side in adjacent segments (Martindale and Shankland, 1990).

Two other unpaired leech neurons have been observed to alternate their side of origin [posteriomedial serotonin (PMS) neuron, Macagno and Stewart, 1987; mz4 neuron, Shankland and Martindale, 1989] and may utilize similar patterning mechanisms. Ablation studies indicate that PMS neuron asymmetry also depends on an interaction between the 2 sides of the embryonic nerve cord (Stuart et al., 1987). However, the RAS and CAS neurons are the only cells for which an interganglionic component of the patterning mechanism has been experimentally demonstrated. It is as yet unclear whether there is a physiological function for the alternation of unpaired neurons in leech embryos, or if this distribution is simply a by-product of the developmental mechanism that shunts right and left cells into alternative developmental pathways. In either case, the intraganglionic interaction would provide a means for producing specialized neurons from otherwise paired, functionally similar cells, and the interganglionic interactions would spatially coordinate these developmental decisions over a span of many segments.

\section{Mechanisms of cell interaction}

How does an AS neuron convey to homologues its own state of commitment with respect to the AS phenotype? The disparate location of the neuronal cell bodies suggests that the interaction occurs via axonal projections, and the morphology of the AS neurons is consistent with this idea. The transmission of a developmental signal between AS homologues could be direct, and indeed, there are reports of short-range neuronal interactions, both synaptic and nonsynaptic, mediating process outgrowth, cell survival, and the determination of cell identity $(\mathrm{Ku}-$ wada and Goodman, 1985; Lipton and Kater, 1989). Alternatively, the interaction of AS homologues could be indirect, requiring the mediation of other cells, as occurs, for instance, during the competition of multiple presynaptic neurons for a limited supply of postsynaptic targets (reviewed in Purves and Lichtman, 1985). Experimental studies provide some support for a direct route of interaction, because both RAS and CAS neuron alternation are largely unaffected by extensive nervous system lesions as long as those lesions do not include a cell lineage giving rise to the AS neuron (Martindale and Shankland, 1990). Nonetheless, many routes of indirect interaction are still plausible.

If CAS neuron homologues communicate between adjacent ganglia via their own axons, it seems likely that the large-caliber primary axon is sufficient for the transmission of interganglionic signals. The fine-caliber secondary axons of the adult CAS neuron are quite variable in their projections to other ganglia, while the degree of alternation between certain ganglion pairs is nearly invariant. However, caution must be exercised when extrapolating from adult cell morphologies, because the interactions responsible for CAS neuron patterning occur during embryonic life. It is clear from antibody-stained specimens that the major process of the embryonic CAS neuron is the anterior contralateral (i.e., primary) axon (Shankland and Martindale, 1989); nonetheless, it is also possible that embryonic CAS neurons have a more constant array of finer-caliber interganglionic axons, some of which may regress as development proceeds (Wallace, 1984; Gao and Macagno, 1987).

Our present experiments indicate that a given CAS neuron can influence neuropeptide expression in anterior and posterior segments, and if we assume that the pertinent developmental signals are transmitted along the primary axon, then those signals must travel in both anterograde and retrograde directions. In the anterograde direction, a CAS neuron homologue would signal its own state of commitment to one or both of its homologues in the next anterior ganglion. In the retrograde direction, the neuron would be able to detect the asymmetry of its homologues in the next anterior ganglion and modulate its own neuropeptide expression accordingly. A similar situation is observed with the RAS neuron, which is able to interact in both directions (Fig. 3), though its large-caliber axon projects posteriorly (Shankland and Martindale, 1989). Bidirectional transmission would be expected if the pertinent signals were electri- 
cal, or if they involve the widely studied anterograde and retrograde transport of macromolecules through the axonal cytoplasm (Grafstcin and Forman, 1980).

Whatever the mechanism of interaction, an AS homologue must be able to distinguish between its ipsilateral and contralateral homologues in at least the 2 adjacent segments. This distinction is unlikely to depend on the recognition of cell surface markers unique to the right and left sides, given that we can experimentally force alternation in either direction. A more reasonable hypothesis is that the interaction of AS neuron homologues is, in large part, constrained by their respective branching patterns. Because CAS axons rarely form crossing branches in anterior ganglia, the axons entering a ganglion through the right and left connectives would encounter a CAS homologue neuron in that segment on distinct portions of its own arborization (Fig. 8). Thus, if the interganglionic interaction depends upon a closerange interaction (e.g., direct synapse formation), the anterior neuron would in effect distinguish which of its 2 homologues in the next posterior ganglion, the nascent CAS neuron or the neuron losing the competition, has contacted its ipsilateral branches, and which has contacted its contralateral branches. In a similar fashion, these axons could transmit information back to their cell bodies regarding the asymmetry of CAS determination in the anterior ganglion. An active interplay between anterior- and posterior-going signals would coordinate the asymmetry of neuronal differentiation in successive segments, and the final pattern of AS neuron differentiation would emerge from this coordinated pattern of cell interactions.

\section{References}

Blair SS (1983) Blastomere ablation and the developmental origin of identified monoamine-containing neurons in the leech. Dev Biol 95: 65-72.

Evans BD, Calabrese RL (1989) SCP-like immunoreactivity and its co-localization with FMRF-amide-like immunoreactivity in the central nervous system of the leech, Hirudo medicinalis. Cell Tissue Res 257:187-199.

Gao W-Q, Macagno ER (1987) Extension and retraction of axonal projections by some developing neurons in the leech depends upon the existence of neighboring homologues. I. The HA cells. J Neurobiol $18: 43-59$.

Gimlich RL, Braun J (1985) Improved fluorescent compounds for tracing cell lineage. Dev Biol 109:509-514.

Grafstcin B, Forman DS (1980) Intracellular transport in neurons. Physiol Rev 60:1167-1283.
Kimble J (1981) Alterations in cell lineage following laser ablation of cells in the somatic gonad of Caenorhabditis elegans. Dev Biol 87: 286-300.

Kramer AP, Weisblat DA (1985) Developmental neural kinship groups in the leech. J Neurosci 5:388-407.

Kuwada JY, Goodman CS (1985) Neuronal determination during embryonic development of the grasshopper nervous system. Dev Biol 110:114-126.

Lipton SA, Kater SB (1989) Neurotransmitter regulation of neuronal outgrowth, plasticity and survival. Trends Neurosci 12:265-270.

Macagno ER, Stewart RR (1987) Cell death during gangliogenesis in the leech: competition leading to the death of PMS neurons has both random and nonrandom components. J Neurosci 7:1911-1918.

Martindale MQ, Shankland M (1990) Neuronal competition determines the spatial pattern of neuropeptide expression by identified neurons in the leech. Dev Biol 139:210-226.

Ort CA, Kristan WB Jr, Stent GS (1974) Neuronal control of swimming in the medicinal leech. II. Identification and connections of motor neurons. J Comp Physiol 94:121-154.

Purves D, Lichtman JW (1985) Principles of neural development. Sunderland, MA: Sinauer.

Shankland M (1984) Positional determination of supernumerary blast cell death in the leech embryo. Nature 307:541-543.

Shankland M, Martindale MQ (1989) Segmental specificity and lateral asymmetry in the differentiation of developmentally homologous neurons during leech embryogenesis. Dev Biol 135:431-448.

Stent GS (1985) The role of cell lineage in development. Phil Trans R Soc Lond [Biol] 312:3-19.

Stent GS, Weisblat DA, Blair SS, Zackson SL (1982) Cell lineage in the development of the leech nervous system. In: Neuronal development (Spitzer NC, ed), pp 1-44. New York: Plenum.

Stuart DK, Blair SS, Weisblat DA (1987) Cell lineage, cell death, and the developmental origin of identified serotonin- and dopamine-containing neurons in the leech. J Neurosci 7:1107-1122.

Sulston JE, White JG (1980) Regulation and cell autonomy during postembryonic development of Caenorhabditis elegans. Dev Biol 78: $577-597$.

Wallace BG (1984) Selective loss of neurites during differentiation of cells in the leech central nervous system. J Comp Neurol 228:149153.

Weisblat DA, Blair SS (1984) Developmental indeterminacy in embryos of the leech Helobdella triserialis. Dev Biol 101:326-335.

Weisblat DA, Shankland M (1985) Cell lineage and segmentation in the leech. Phil Trans R Soc Lond [Biol] 312:39-56.

Weisblat DA, Harper G, Stent GS, Sawyer RT (1980) Embryonic cell lineage in the nervous system of the glossiphoniid leech Helobdella triserialis. Dev Biol 76:58-78.

Whitman CO (1878) The embryology of Clepsine. J Microsc Sci 18: 215-315. 